\title{
Prognostic and Remaining Life Prediction of Electronic Device under Vibration Condition Based on CPSD of MPI
}

\author{
Ying Chen, Ning Tang, and Zenghui Yuan \\ Science and Technology on Reliability and Environmental Engineering Laboratory, Reliability and System Engineering Department, \\ Beihang University, Beijing 100191, China \\ Correspondence should be addressed to Ning Tang; 632057638@qq.com
}

Received 22 July 2016; Revised 29 September 2016; Accepted 20 October 2016

Academic Editor: Minvydas Ragulskis

Copyright (C) 2016 Ying Chen et al. This is an open access article distributed under the Creative Commons Attribution License, which permits unrestricted use, distribution, and reproduction in any medium, provided the original work is properly cited.

\begin{abstract}
Prognostic of electronic device under vibration condition can help to get information to assist in condition-based maintenance and reduce life-cycle cost. A prognostic and remaining life prediction method for electronic devices under random vibration condition is proposed. Vibration response is measured and monitored with acceleration sensor and OMA parameters, including vibration resonance frequency, especially first-order resonance frequency, and damping ratio is calculated with cross-power spectrum density (CPSD) method and modal parameter identification (MPI) algorithm. Steinberg vibration fatigue model which considers transmissibility factor is used to predict the remaining life of electronic component. Case study with a test board is carried out and remaining life is predicted. Results show that with this method the vibration response characteristic can be monitored and predicted.
\end{abstract}

\section{Introduction}

Prognostics and Health Monitoring (PHM) of electronic devices integrates sensor data with models that enable assessment of the degradation of a product from an expected normal operating condition and also assesses the future reliability of the product based on current and historic conditions [1]. The technique is intended for condition monitoring in high reliability applications where the knowledge of impending failure is critical and the risks in terms of loss-of-functionality are too high to bear. In avionics and aerospace electronics systems, electronic components, and dynamic loading are applied to electronic devices during normal operation, which includes high temperature and temperature cycling, vibration, and humidity. Data show that $20 \%$ [1] of electronic equipment failure is related to vibration and shock. PHM of electronic under vibration condition can help electronic devices to meet the safety, reliability, maintainability, and supportability requirements and reduce life-cycle cost.

PHM of electronic devices under vibration condition has been studied by some researchers, which is mainly based on two kinds of method, data-based method and model-based method. Lall et al. developed a statistical method for prognostication of area-array electronics under shock and vibration loads during vibration testing, which is based on state space vectors from resistance spectroscopy measurements [2]. Kalman filter method is used to estimate the state space feature vector, extrapolate the future state, and predict the remaining useful life. Results will help to schedule downtime for maintenance and select the appropriate time to reorder a replacement part. Aiming at the phenomenon that the recent studies which predict impending failures of power electronic components' packaging are not explicit, they proposed monitor system for ceramic area-array-package with resistance spectroscopy and high speed cameras, predicted with system-transfer function and statistical pattern recognition techniques during shock test [3]. Results show that there is degradation in statistical confidence values, and the impending failure may have been detected before failure of the electronic assembly.

Compared with data-based prognostic method, modelbased method provides the relation between failure and the characteristic of product, which helps to have the proper knowledge of failures and take measures to prevent them. $\mathrm{Gu}$ et al. proposed a model-based health monitoring and 
prognostics method for assessing the reliability of a printed circuit board (PCB) under random vibration by using strain gauges and accelerometer to measure the vibration response [1]. The Physics-of-Failure fatigue model and Miner accumulation rule are used to assess the remaining life of a test board. Modal analysis with FEA software is conducted to transfer global sensor information to local sensor information. Miner rule is used to accumulate the damage of different loading condition. An uncertainty analysis of prognostics of electronics subject to random vibration, which includes measurement uncertainty, parameter uncertainty, failure criteria uncertainty, and future usage uncertainty, is also conducted [4]. A case study is presented whereby prognostics with uncertainty are applied to an electronic circuit board subject to random vibration. Gucik-Derigny et al. presented a modelbased prognosis approach for assessing the remaining useful life of a PCB subject to low frequency thermal fatigue damage and high frequency of vibration damage [5]. The proposed prognosis method was also divided into fast dynamics part which includes the estimation of nonmeasurable state and slowly drifting PCB damping parameter and slow dynamic part which includes estimation of low cycle fatigue BGA solder joint interconnection damage state and parameter. Simulation is done to test the efficiency of their approach.

For model-based prognosis approach, modal parameters, such as frequency and damping, are associated with the prediction of remaining useful life, which will also change during the process of work. Recent research evaluates these parameters by simulation with FEA or CalcePWA software, which may add offline procedures and may need more experience of skilled person. Online measurement of these parameters and using them properly in the prediction model is critical. Operational Modal Analysis (OMA) technique can determine the inherent properties of a structure by measuring only the response of it without using an artificial excitation. Modal parameter identification is the technique to calculate the resonance frequencies, damping, and mode shapes of a structure. In OMA all modal parameters are to be determined without knowing the excitation forces. Therefore it is normally assumed that the excitation forces are Gaussian white noise [6]; recent research has extended the excitation to harmonic excitation [7], nonstationary excitation [8], and periodic excitations [9]. OMA has widely been used in performance assessment of structure whose properties would change with time [10-12], and recently the researchers have raised the attention on health diagnosis of structure and estimating the remaining service life in civil engineering [13] and oil industry [14].

The research on physics model of vibration fatigue failure can be traced back to the 1970s. After many years of practical experience, Steinberg proposed Steinberg model applied to lifetime estimation of electronic devices working under sinusoidal or random vibration conditions $[15,16]$. Although Manson model and other models appeared in this field later, Steinberg model is still wildly used in engineering for its obvious physical meaning [17]. Chesné and Deraemaeker studied the application of Steinberg model in tantalum capacitor [18]. Through experiment, they provided the S-N curve in different sinusoidal sweeping-frequency vibration conditions and compared the experimental result with Finite Element Analysis (FEA) simulation, with the aim of determining parameter values of Steinberg model. Steenackers et al. proposed some principles of electronic systems to withstand high vibration and shock conditions [19]. Lim analyzed PCB's vibration with Steinberg model in CalcePWA [20]. Yang et al. studied the impact of transmissibility factor of Steinberg model on fatigue lifetime of electronic devices [21], which represent the coupling state of PCB and its case. Neglecting this factor will result in the inaccuracy of prediction.

From the above discussion, previous model based prognostic methods of electronic device under vibration condition are limited, for example, the methods lack online measurement and calculation of model parameters. In this paper, an online health prognostic method for electronic devices under random vibration condition is proposed. Vibration response is measured and monitored with acceleration sensor under white noise random excitation, and OMA parameters, including vibration resonance frequency, especially firstorder resonance frequency, and damping ratio, are calculated with cross-power spectrum density (CPSD) method and modal parameter identification (MPI) algorithm. Steinberg vibration fatigue model which considers transmissibility factor is used to predict the remaining life of electronic component.

The remainder of the paper is organized as follows. Section 2 presents the PHM flowchart with detail steps. Section 3 introduces the cross-power spectrum density (CPSD) method and modal parameter identification (MPI) algorithm. Section 4 discusses the Steinberg model with transmissibility factor. Section 5 illuminates the real case with a test board. Section 6 gives conclusions as well as directions for the future work.

\section{PHM Methodology for Electronics under Vibration Loading}

The proposed health prognostic method for electronics device under random vibration is shown in Figure 1, in which either the vibration response is monitored and analyzed or the remaining life is predicted online.

The first step is to select the sensor location on the PCB and prepare the monitoring devices. Since some complicated PCBs have hundreds of components, the space is limited; it is essential to know where to place the sensors. Sensors will be placed at the site where components are most likely to fail. The vibration result of the PCB under given random vibration condition could be obtained. For example, CalcePWA software [1] can identify the natural frequency of the PCB and the locations of critical components at certain vibration loading levels. Piezoelectric sensor is used to measure the time-domain acceleration response of PCB. After that the cross spectrum of signal from different sensor is calculated, results will be used to generate the CPSD of reference point and measurement point. With frequency domain, modal extraction method calculates the first-order resonant frequency and damping of the $\mathrm{PCB}$, which are the main parameters needed to calculate the remaining life time with PoF models. During the process, monitor the 

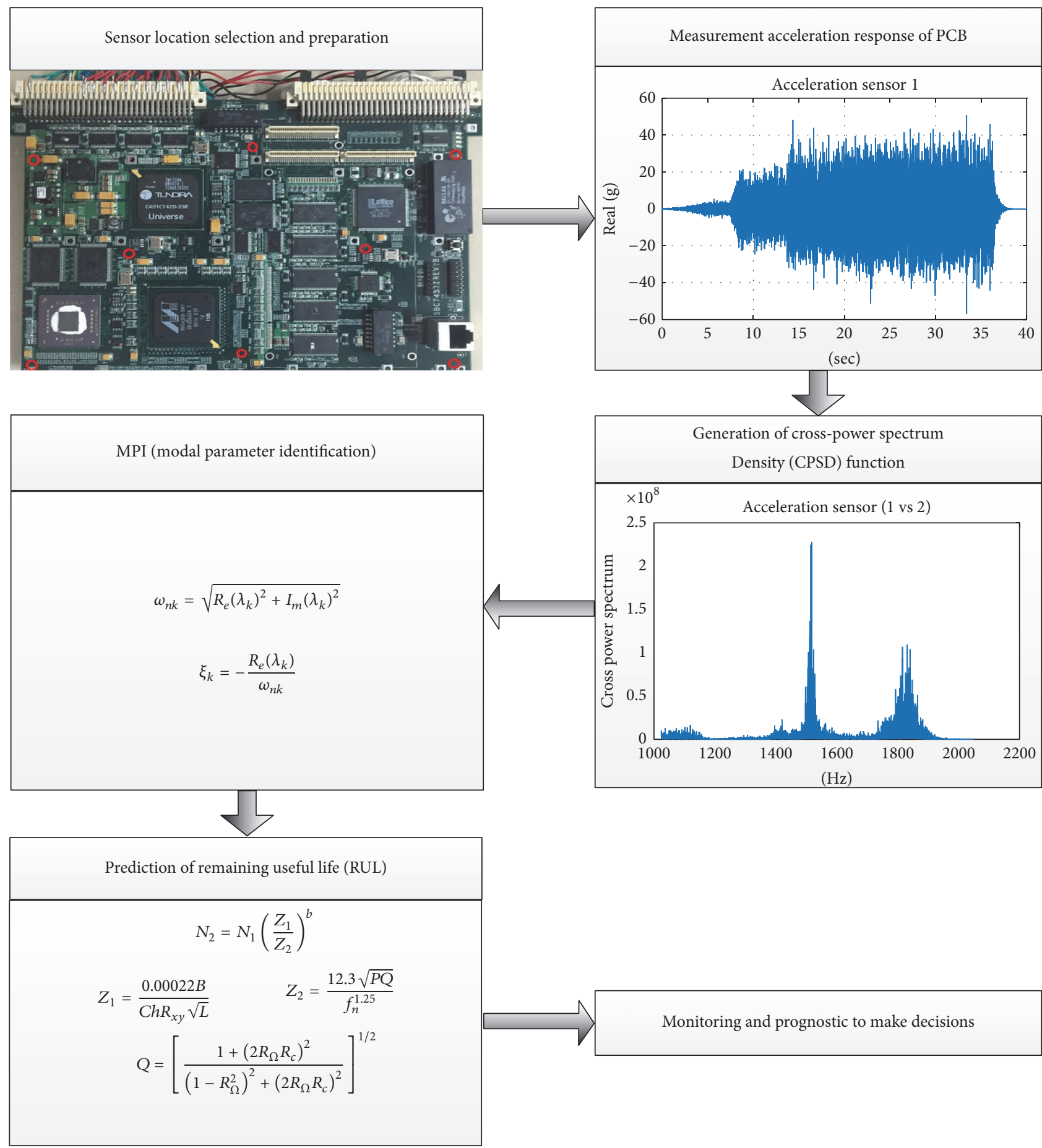

FIGURE 1: Proposed prognostic method of electronic devices under vibration condition.

vibration response signals and the modal parameters and make decisions when necessary.

\section{CPSD Based Modal Parameter Identification}

3.1. Cross-Power Spectrum Density Function. Piezoelectric acceleration sensor can measure the system response in time domain and Discrete Fourier Transform (DFT) should be carried out to obtain the power spectral density matrices that will contain all the frequency information. The relationship between the input $x(t)$ and the output $y(t)$ in frequency domain can be written in the following form:

$$
\left[G_{y y}(j \omega)\right]=[H(j \omega)]^{*}\left[G_{x x}(j \omega)\right][H(j \omega)]^{T},
$$


where $G_{x x}(j \omega)$ is the input power spectral density matrix that is constant in the case of a stationary zero mean white noise input. This constant will be called $C$ in the rest of the mathematical derivation. $G_{y y}(j \omega)$ is the output PSD matrix and $H(j \omega)$ is the frequency response function (FRF) matrix. As seen in (1), the output $G_{y y}$ will be highly sensitive to the input constant $C$. The rest of the equation derivations and single degree of freedom identification will provide relevant results, only by assuming that the input is effectively represented by a constant value (mean Gaussian). It is therefore important to realize how this input assumption will be crucial to the technique.

The FRF matrix can be written in a typical partial fraction form (used in classical modal analysis), in terms of poles and residues where $\left[G_{x x}(\omega)\right]$ is the input power spectral density (PSD) matrix, $\left[G_{y y}(\omega)\right]$ is the output PSD matrix, and $[H(\omega)]$ is the frequency response function (FRF) matrix, and * and superscript $T$ denote complex conjugate and transpose, respectively. The FRF matrix can be written in a typical partial fraction form (used in classical Modal analysis), in terms of poles, $\lambda$, and residues, $R$, which is

$$
\begin{aligned}
{[H(\omega)] } & =\sum_{k=1}^{N} \frac{\left[R_{k}\right]}{j \omega-\lambda_{k}}+\frac{\left[R_{k}\right]^{*}}{j \omega-\lambda_{k}^{*}}, \\
\lambda_{k} & =-\xi_{k}+j \omega_{n k},
\end{aligned}
$$

where $N$ is the total number of modes of interest, $\lambda_{k}$ the pole of the $k$ th mode, $\xi_{k}$ the modal damping (decay constant), and $\omega_{n k}$ the damped natural frequency of the $k$ th mode.

$$
\omega_{d k}=\omega_{0 k} \sqrt{1-\varsigma_{k}^{2}}=\omega_{0 k} \sqrt{1-\frac{\sigma_{k}^{2}}{\omega_{0 k}}}
$$

$\varsigma_{k}$ being the critical damping and $\omega_{0 k}$ the undamped natural frequency, both for mode $k$.

The transfer function matrix $[H]$ is symmetric and an element $H_{p q}(j \omega)$ of this matrix is then written in terms of the component $r_{k p q}(j \omega)$ of the residue matrix as follows:

$$
H_{p q}(j \omega)=\sum_{k=1}^{N} \frac{r_{k}(p, q)}{j \omega-\lambda_{k}}+\frac{r_{k}(p, q)^{*}}{j \omega-\lambda_{k}^{*}} .
$$

Using expression (1) for the matrix $\left[G_{y y}(\omega)\right]$ and the Heaviside partial fraction theorem for polynomial expansions, the following expression for the output PSD matrix is $\left[G_{y y}(\omega)\right]$ assuming the input is random in both time and space and has a zero mean white noise distribution; that is, $\left[G_{x x}(\omega)\right]=[C]$ :

$$
\begin{aligned}
& G_{y y}(j \omega)=\sum_{k=1}^{N}\left(\frac{\left[A_{k}\right]}{j \omega-\lambda_{k}}+\frac{\left[A_{k}\right]^{*}}{j \omega-\lambda_{k}^{*}}+\frac{\left[B_{k}\right]}{-j \omega-\lambda_{k}}\right. \\
& \left.+\frac{\left[B_{k}\right]^{*}}{-j \omega-\lambda_{k}^{*}}\right)
\end{aligned}
$$

where $\left[A_{k}\right]$ is the $k$ th residue matrix of the matrix $\left[G_{y y}\right]$. The matrix $G_{x x}$ is assumed to be a constant value $C$, since the excitation signals are assumed to be uncorrelated zero mean white noise in all the measured DOFs.

In (5), the form of $G_{y y}(\omega)$ is similar to frequency response function, the poles also including frequency and damping information. By replacing the traditional frequency response function with cross-power spectrum density function, it is possible to identify working modal parameters.

Cross-power spectrum is the product of Fourier transforms of test response signal and the reference response signal. Its amplitude is the product of the amplitude of test response signal and the reference. Its phase is the difference of the phase of test response signal and the reference. The relations between FRF and cross-power spectrum and working modal parameter identification algorithm based on crosspower spectrum will be demonstrated at the next section.

3.2. Modal Parameter Identification Algorithm. For a system with $m$ measurement point and $p$ reference point, the crosspower spectrum density matrix is

$$
G(j \omega)=\left[\begin{array}{cccc}
G_{11}(j \omega) & G_{12}(j \omega) & \cdots & G_{1 P}(j \omega) \\
G_{21}(j \omega) & G_{22}(j \omega) & \cdots & G_{2 P}(j \omega) \\
\vdots & \vdots & \vdots & \vdots \\
G_{M 1}(j \omega) & G_{M 2}(j \omega) & \cdots & G_{M P}(j \omega)
\end{array}\right]_{M \times P}
$$

and then (5) can be written as

$$
\begin{gathered}
G_{y y}(j \omega)=\sum_{k=1}^{N}\left(\frac{\left\{\psi_{k}\right\}\left\{W_{k}\right\}^{T}}{j \omega-\lambda_{k}}+\frac{\left\{\psi_{k}\right\}^{*}\left\{W_{k}\right\}^{H}}{j \omega-\lambda_{k}^{*}}\right. \\
\left.+\frac{\left\{W_{k}\right\}\left\{\psi_{k}\right\}^{T}}{-j \omega-\lambda_{k}}+\frac{\left\{W_{k}\right\}^{*}\left\{\psi_{k}\right\}^{H}}{-j \omega-\lambda_{k}^{*}}\right)
\end{gathered}
$$

in which $\left\{\psi_{k}\right\},\left\{\psi_{k}\right\}^{*},\left\{W_{k}\right\}$, and $\left\{W_{k}\right\}^{*}$ are complex modal matrix.

$$
\begin{aligned}
& \text { Let } s=j \omega, O(s)=(S I-Z)^{-1} W \text {, and (6) can be written as } \\
& \qquad G(s)=\phi(S I-Z)^{-1} W=\phi O(s) .
\end{aligned}
$$

The time derivative of $G(s)$ is

$$
\begin{aligned}
G(s) & =S G(s)-R(0)=S G(s)-\phi W \\
& =S \phi O(s)-\phi(S I-Z) O(s)=\phi Z O(s)
\end{aligned}
$$

which will get

$$
\begin{aligned}
{\left[\begin{array}{l}
G(s) \\
G(s)
\end{array}\right] } & =\left[\begin{array}{c}
\phi \\
\phi Z
\end{array}\right] O(s) \\
\text { or }\left[\begin{array}{c}
G(s) \\
S G(s)
\end{array}\right]-\left[\begin{array}{c}
0 \\
\phi W
\end{array}\right] & =\left[\begin{array}{c}
\phi \\
\phi Z
\end{array}\right] O(s) .
\end{aligned}
$$

For Eigenvalue matrix and feature vector matrix, there must be a matrix, which will fulfill the Eigenvalue function of

$$
A \phi+\phi \mathrm{Z}=0 .
$$


Another form of (9) is

$$
[A \vdots I]\left[\begin{array}{c}
\phi \\
\phi Z
\end{array}\right]=0 .
$$

Multiply $O(s)$ to (10) and get

$$
\begin{aligned}
{[A \vdots I]\left[\begin{array}{c}
\phi \\
\phi Z
\end{array}\right] O(s) } & =[A \vdots I]\left[\begin{array}{c}
G(s) \\
S G(s)-\phi W
\end{array}\right]=0, \\
A G(s)+S G(s)-\phi W & =0
\end{aligned}
$$

Consider $S=j \omega$; then (12) can be given by

$$
A G\left(j \omega_{k}\right)+j \omega_{k} G\left(j \omega_{k}\right)=\phi W, \quad k=1,2, \ldots, K .
$$

Let

$$
\begin{aligned}
& D=\left[G\left(j \omega_{1}\right), G\left(j \omega_{2}\right), \ldots, G\left(j \omega_{1 K}\right)\right], \\
& \Omega=-\operatorname{diag}\left[j \omega_{1} I, j \omega_{2} I, \ldots, j \omega_{k} I\right]
\end{aligned}
$$

and then

$$
[A \vdots-\phi W]\left[\begin{array}{c}
D \\
{\left[I_{1}, I_{2}, \ldots, I_{k}\right]}
\end{array}\right]=D \Omega .
$$

$D$ and $\Omega$ are all functions of measured CPSD and $\omega_{i}$, which are known; the matrix $A$ and $\phi W$ can be solved by (14):

$$
\begin{aligned}
\text { Modal frequency: } \omega_{n k} & =\sqrt{R_{e}\left(\lambda_{k}\right)^{2}+I_{m}\left(\lambda_{k}\right)^{2}} \\
\text { Damping } \xi_{k} & =-\frac{R_{e}\left(\lambda_{k}\right)}{\omega_{n k}}, \quad n=1, \ldots, N .
\end{aligned}
$$

\section{Remaining Useful Life Prediction}

Steinberg model is used for remaining useful life prediction, which is derived from component fatigue characteristic fitting from fatigue experiments data [22], where

$$
N_{1} S_{1}^{b}=N_{2} S_{2}^{b}
$$

in which $N_{1}$ and $N_{2}$ are the stress cycle before fatigue. $S_{1}$ and $S_{2}$ are the stress when failure happens, and $b$ is the fatigue index number related to the linear gradient of fatigue curve. For linear system, stress $S$ is proportionate to displacement $Z$, and (19) can be written as

$$
\begin{aligned}
N_{1} Z_{1}^{b} & =N_{2} Z_{2}^{b}, \\
N_{2} & =N_{1}\left(\frac{Z_{1}}{Z_{2}}\right)^{b} .
\end{aligned}
$$

Under vibration condition, the displacement of simply supported PCB with four sides can expressed as follows, where the fatigue lifetime of these component can reach $1 \times$ $10^{7}$ stress cycles [22]:

$$
Z_{1}=\frac{0.00022 B}{C h R_{x y} \sqrt{L}}
$$

where $B$ is the length of the circuit board edge parallel to the component, $L$ is the length of the electronic component, $h$ is the circuit board thickness, $C$ is the constant for different types of electronic components $(0.75<C<2.25$, and refer to [23]), and $R_{x y}$ is the relative position factor for the component mounted on the board.

$$
R_{x y}=\sin \frac{\pi X}{a} \sin \frac{\pi Y}{b},
$$

where $X$ and $Y$ are horizontal and longitudinal position of the component and $a$ and $b$ are length and width of PCB.

Based on the vibration fatigue curve, incorporating the system dynamic response property analysis, Steinberg proposed a vibration fatigue model for electronic device. In his model, PCB can be approximated as a single degree of freedom system, when it vibrates under the fundamental resonance.

When the input power spectral density (PSD) of random vibration is flat spectrum in resonance region, the mean square root acceleration response of a system is given by

$$
G_{\text {out }}=\sqrt{\frac{\pi}{2} P f_{n} Q}(\mathrm{RMS}) \text {. }
$$

The actual dynamic single amplitude displacement of PCB's center is given by [15]

$$
Z_{2}=\frac{9.8 G_{\text {out }}}{f_{n}^{2}}=\frac{12.3 \sqrt{P Q}}{f_{n}^{1.25}},
$$

where $P$ is the input PSD at resonant frequency, $f_{n}$ is resonant frequency, and $Q$ is the transmissibility at resonant frequency, which is given by [15]

$$
\begin{aligned}
Q & =\left[\frac{1+\left(2 R_{\Omega} R_{c}\right)^{2}}{\left(1-R_{\Omega}^{2}\right)^{2}+\left(2 R_{\Omega} R_{c}\right)^{2}}\right]^{1 / 2}, \\
R_{\Omega} & =\frac{\omega}{\omega_{n}}, \\
R_{c} & =\frac{\xi_{1}}{\xi_{c}}
\end{aligned}
$$

where $\omega_{n}=\sqrt{K / m}$, being the first-order angular resonance frequency, $K$ is rigidity, $m$ is mass, $\xi_{1}$ is the damping at firstorder frequency, and

$$
\xi_{c}=2 \sqrt{K m}
$$

The $f_{n}$ and damping ratio is measured and calculated using (18).

\section{Case Study}

5.1. The Test Board. A certain type of test board (shown in Figure 2) is mainly used for ground automatic control systems. There are CPU microprocessor, memory and cache, sensor chips, counters, and various interfaces on the single board, and its function is like a microcomputer. There are 


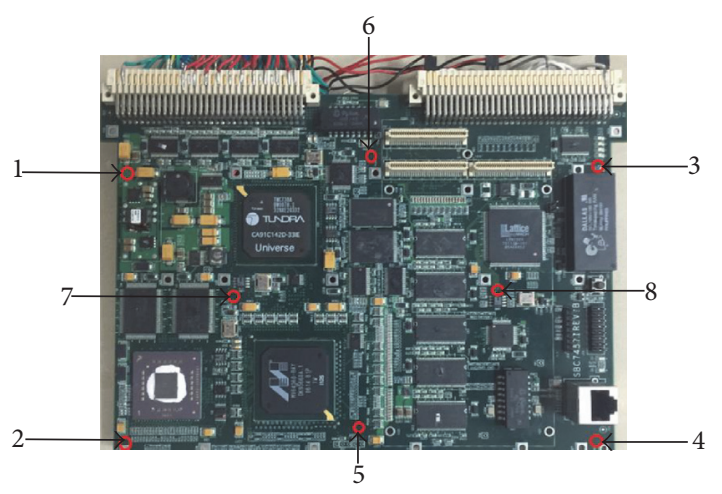

FIGURE 2: Test board and acceleration sensor layout.

many types of components on the test board including integrated circuits, chip capacitors, tantalum capacitors, metalfilm resistors, inductors, connectors, crystals, switches, and PCB (including the solder joint, pad, PTH, vias, and metal interconnects). The material of PCB is FR-4; material of metal wires and plated through holes (PTH) is copper, and solder is $63 \mathrm{Sn} 37 \mathrm{~Pb}$. The main types of package of the device are BGA, SOP, QFP, DIP, TSSOP, and so forth. And packaging materials are mainly plastic or ceramic. PCB is mounted through the holes on its four edges side, and it is considered that the installation site will not fail.

5.2. The Monitoring Devices. The monitoring system is shown in Figure 3, which mainly includes the vibration table (LDS), acceleration sensor, vibration measurement instrument, and data collection and analysis software. The working frequency of LDS vibration table is $5-4000 \mathrm{~Hz}$, and vibration table can vibrate horizontally and vertically. The payload weight of the vibration table is about $200 \mathrm{Kg}$. Vibration Signal Control System is the brain of LDS vibration table, which has the function of random and sinusoidal vibration, harmony frequency lingering, and shock wave controlling. The vibration signal should be amplified by a power amplifier before sent to the vibration table. Endevco 2222c piezoelectric sensor whose weight is only 2 grams is used to measure the acceleration response of PCB. Vibration measurement instrument is DP730 24-channel device which could analyze polytype of vibration and noise in real time with software. The monitoring system is composed of data collection module, modal parameter identification module, and RUL calculation module.

The test board was mounted on vibration table, and its two edges were clamped, as shown in Figure 3. Random vibration loading condition with a frequency from 10 to $2000 \mathrm{~Hz}$ is applied to the vibration table, which is shown in Figure 4. Vibration spectrum is given by LDS vibration control system and the signal is exported into a power amplifier to drive the vibration table. The vibration table would generate vibration in the vertical direction.

There are mainly four ways to connect sensors and the test object, by bolt, adhesives, wax, or permanent magnet. The
Endevco 7703A-50 acceleration sensor in this test is with no magnetic base and requires high upper limited frequency. So we fix them via hard adhesives. Theoretically, more acceleration sensors can improve the measurement and prediction accuracy. But restricted to the space of PCB, the number of sensors is limited. This may be better if wireless and built-in sensors are used. In our case study, the layout of the test board is very compact and most of the components are on the front of the PCB. Modal analysis and random vibration analysis are carried out with CalcePWA. Results show the displacement response of this test board is well-distributed. Based on these pieces of information, we arranged eight acceleration sensors on the well-distributed eight locations where there is no component to avoid interaction between them.

The broadband random vibration spectrum is set close to white noise and loaded on the vibration table along the normal direction of the PCB. Via the fixture, excitation signal will be transmitted from the vibration table to the electronic equipment.

5.3. Prognostic Data and Remaining Life Prediction. The acceleration data were collected by the DP730 vibration instrument. Meanwhile, the acceleration data was sent to the data collection module which sample and record the data in real time. The modal parameter identification module will identify working modal parameters including the natural frequency and damping of the test board in accordance with the cross-power spectrum. Figure 4 is the exaction vibration spectrum, which is a white noise spectrum. Figure 5 shows the time-domain data of the measurement points 1 to 8 , which express the dynamic response of the PCB. Choose point 1 as the reference point. Figure 6 is the cross-power spectrum density curve of points 2-8 with reference point 1 .

The times series data could be processed using the Fourier transform method. Multiplying the reference point's Fourier transform data by the conjugation of other measuring points, the cross-power spectrum could be obtained, which illustrate the correlation of the two-channel vibrate data. The cross-power spectrum density curves in Figure 6 indicate the correlation between the monitoring point and reference point. PCB is mounted through the holes on its four edges side. Monitoring points 1-6 are located around the PCB, but points 7 and 8 are located in the center of the PCB. So the curves' shape between monitoring points 2-6 and point 1 is consistent, and similarly, the curves' shape between monitoring points $2-6$ and point 1 is consistent. Due to the transmission delay of the vibration signal on the board, the first curves and the last two curves are different. The transmission delay of the vibration signal has an effect on the cross-power spectrum density curves. We can find that the vibration magnitude of output signal is more dependent on the input signal when the frequency component of input signal approach $1550 \mathrm{~Hz}$ or $1850 \mathrm{~Hz}$. The resonance frequency is calculated based on the modal parameter identification algorithm according to the first six curves. The peaks in Figure 6 indicate that the vibration data of other measuring point has significant correlation with the reference point. 


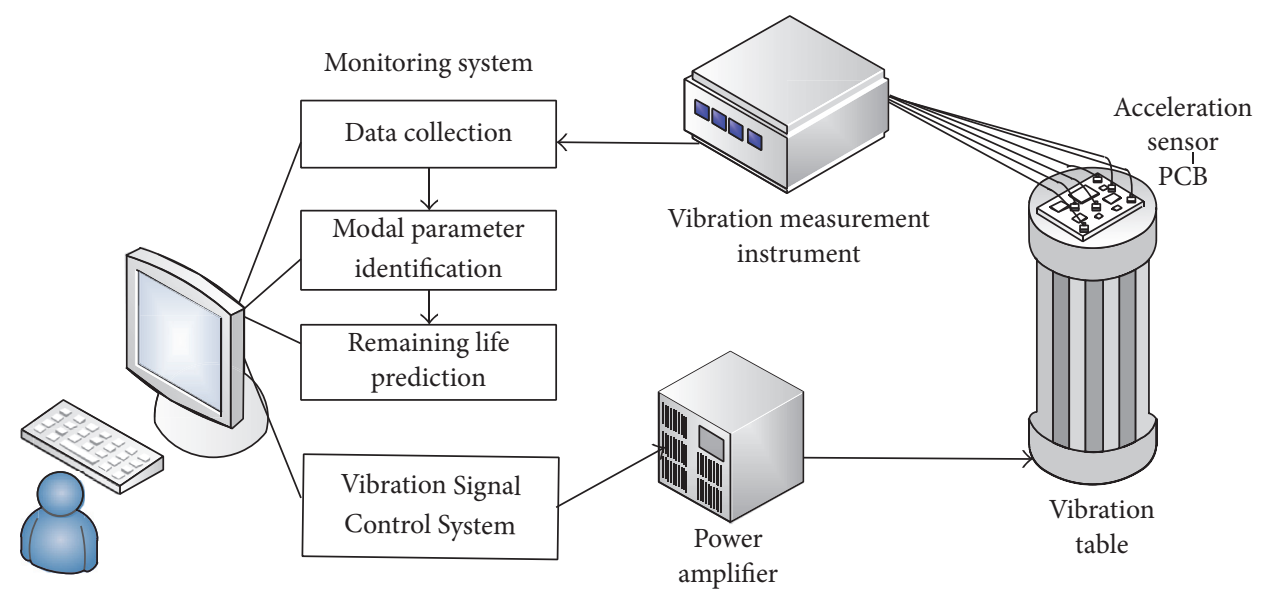

FIGURE 3: Monitoring devices of electronic devices under vibration.

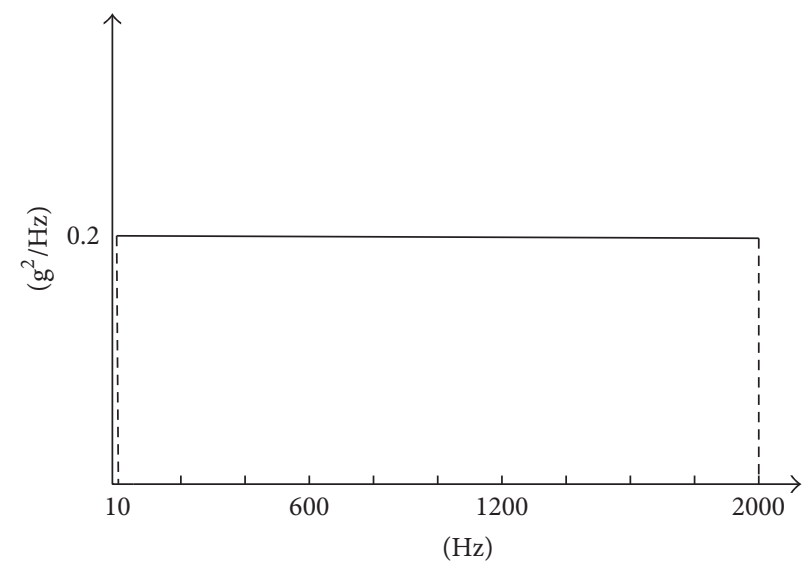

FIGURE 4: Exaction vibration spectrum, a white noise spectrum.

Through the identification of modal parameters, the first three work mode shapes of the PCB board would be obtained. Modal analysis and random vibration analysis are carried out with CalcePWA. The modal analysis results of cross-power spectrum estimation and the modal analysis results of the CalcePWA are consistent.

The remaining useful life of some important component predicted with (20)-(25) is presented in Table 1, in addition of the estimated first-order modal frequency, damping ratio at the beginning of the monitor $(t=0)$, and when $t=$ 28 days, $t=63$ days, and $t=121$ days the data are also given. It can be seen from the results that, without consideration of measurement and computation deviation, the first-order modal frequency of the test board is not significantly changed; the damping will increase with the time.

Table 1 shows that the prediction for four components. All but BGA64 of the components lasted more than one year. BGA64 and QFP100, which have large dimension and are located at the center of the test board, where the vibration response is strong than other components, have less lifetime.

\section{Discussion and Conclusion}

This paper presents an online health prognostic and monitoring method for assessing the reliability of electronic devices under random vibration condition, using acceleration sensor's information measured on the place where there are some critical components and the acceleration sensor could be placed perfectly. Based on cross-power spectrum density (CPSD) method and modal parameter identification (MPI) algorithm, the relationship between modal parameter and the remaining life is established. In the case study, we can assess the reliability of electronic devices without modal analysis and other simulation using software. As a result, this paper proved that an accelerometer is suitable for PHM and makes the field application of PHM much easier, since its 

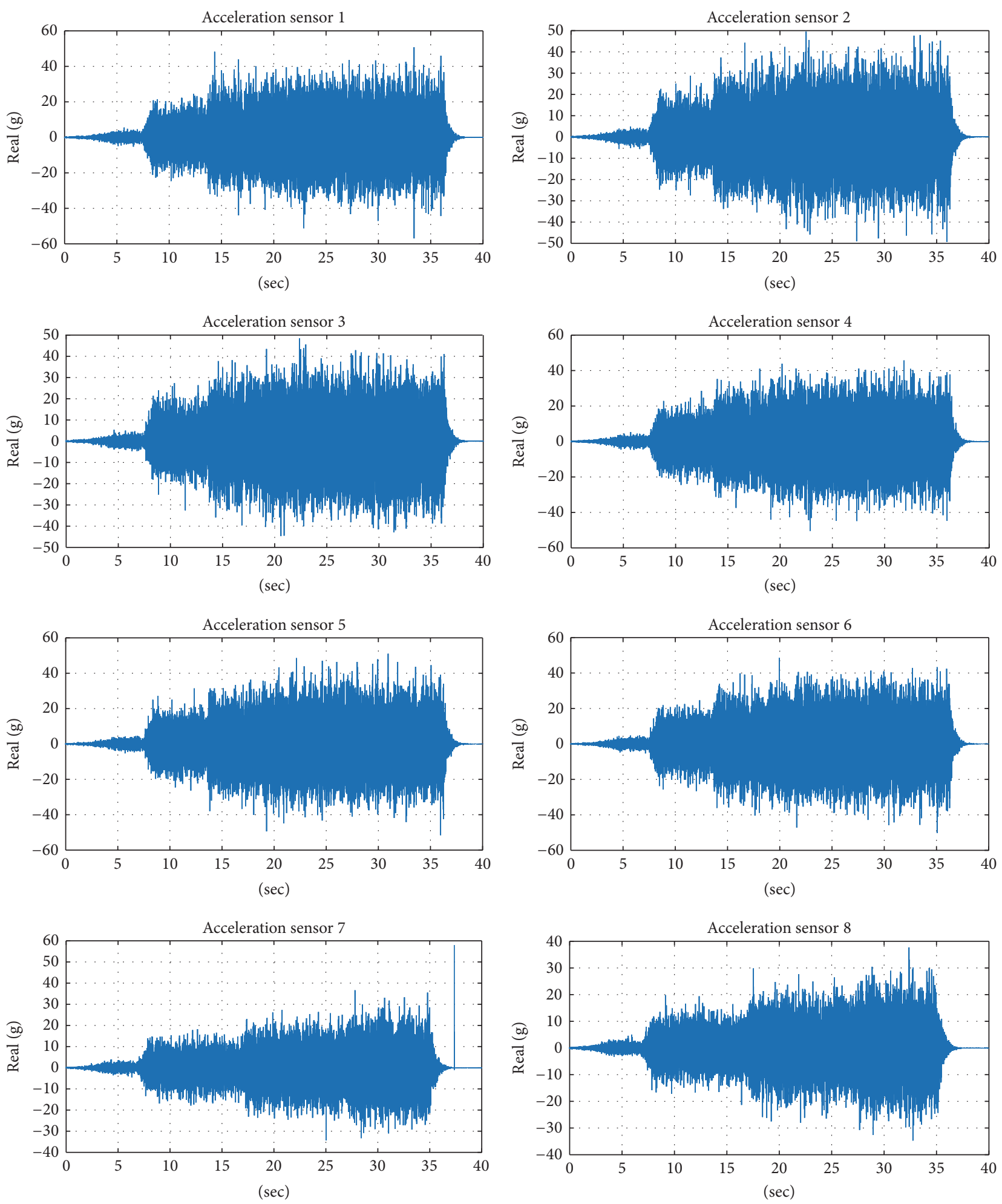

FIGURE 5: Time-domain dynamic response of measurement points 1 to 8 . 

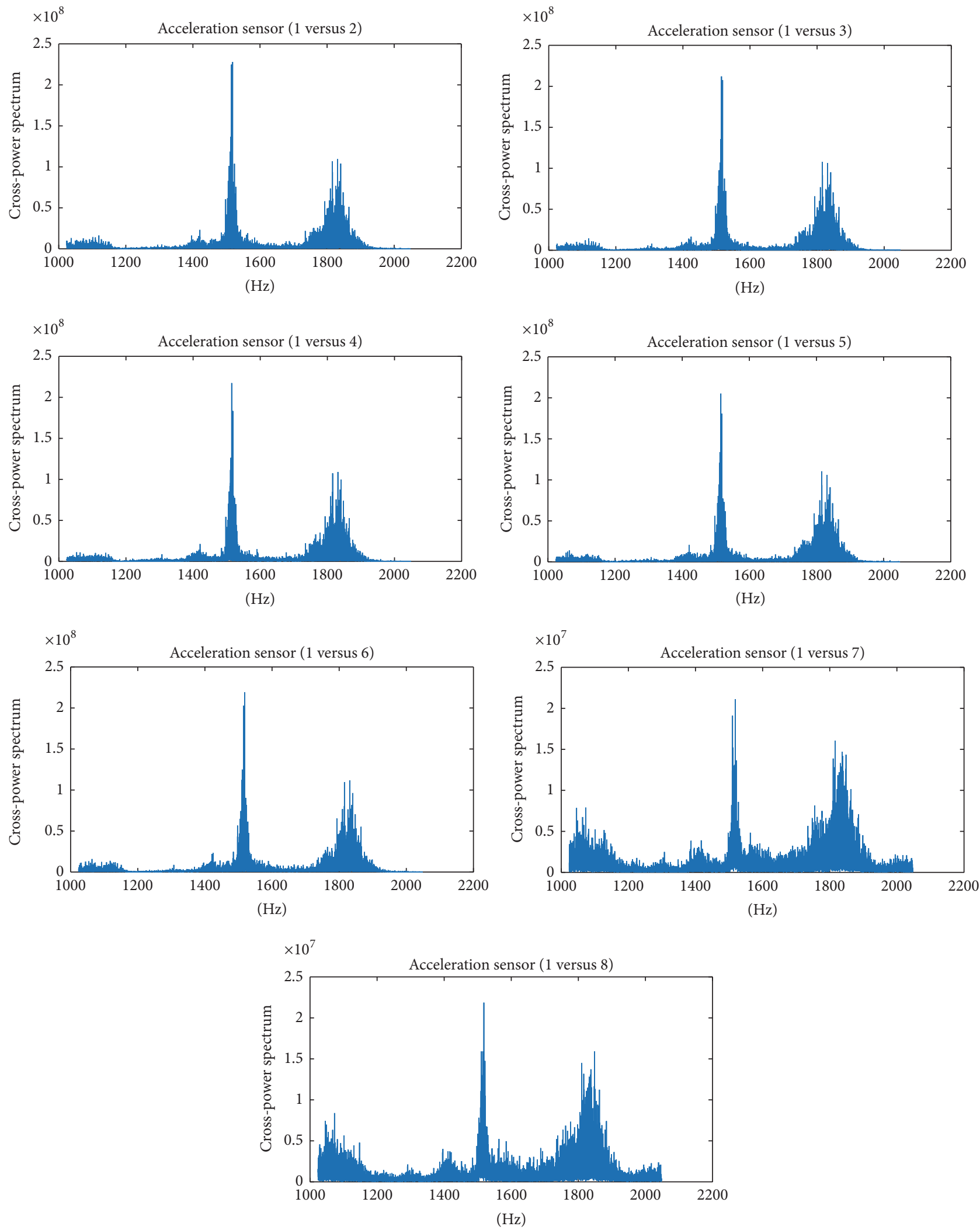

FIgure 6: Cross-power spectrum density curve. 
TABLE 1: Remaining life prediction results.

\begin{tabular}{|c|c|c|c|c|}
\hline Component & $\begin{array}{l}\text { Time } \\
\text { (days) }\end{array}$ & First-order modal Frequency & Damping & $\begin{array}{c}\text { Remaining life prediction } \\
\text { (days) }\end{array}$ \\
\hline \multirow{4}{*}{ BGA64 } & $t=0$ & 231.97 & 3.69 & 332.15 \\
\hline & $t=28$ & 227.41 & 3.73 & 298.73 \\
\hline & $t=63$ & 222.76 & 3.76 & 220.67 \\
\hline & $t=121$ & 220.32 & 3.78 & 183.32 \\
\hline \multirow{4}{*}{ QFP100 } & $t=0$ & 231.97 & 3.69 & 384.29 \\
\hline & $t=28$ & 227.41 & 3.73 & 349.50 \\
\hline & $t=63$ & 222.76 & 3.76 & 281.25 \\
\hline & $t=121$ & 220.32 & 3.78 & 249.66 \\
\hline \multirow{4}{*}{ QFP48 } & $t=0$ & 231.97 & 3.69 & 435.77 \\
\hline & $t=28$ & 227.41 & 3.73 & 405.41 \\
\hline & $t=63$ & 222.76 & 3.76 & 330.92 \\
\hline & $t=121$ & 220.32 & 3.78 & 387.45 \\
\hline \multirow{4}{*}{ SOP32 } & $t=0$ & 231.97 & 3.69 & 592.86 \\
\hline & $t=28$ & 227.41 & 3.73 & 563.43 \\
\hline & $t=63$ & 222.76 & 3.76 & 478.32 \\
\hline & $t=121$ & 220.32 & 3.78 & 449.66 \\
\hline
\end{tabular}

installation and associated signal conditioning circuitry are simpler, cheaper, and more reliable.

Future work should focus on increasing PHM accuracy, by using wireless sensor systems to enhance prediction capability and accuracy by developing or application of algorithms for other vibration excitation, for example, nonwhite noise spectrum.

\section{Competing Interests}

The authors declare that they have no competing interests.

\section{Acknowledgments}

The authors acknowledge the support for the work presented in this paper from National Natural Science Foundation of China (Grant nos. 61503014 and 61573043).

\section{References}

[1] J. Gu, D. Barker, and M. Pecht, "Health monitoring and prognostics of electronics subject to vibration load conditions," Sensors Journal, vol. 9, no. 11, pp. 1479-1485, 2009.

[2] P. Lall, R. Lowe, and K. Goebel, "Prognostics using KalmanFilter models and metrics for risk assessment in BGAs under shock and vibration loads," in Proceedings of the 60th Electronic Components and Technology Conference (ECTC '10), pp. 889901, June 2010.

[3] P. Lall, R. Lowe, and K. Goebel, "Use of prognostics in riskbased decision making for BGAs under shock and vibration loads", in Proceedings of the 12th IEEE Intersociety Conference on Thermal and Thermomechanical Phenomena in Electronic Systems (ITherm '10), pp. 1-11, IEEE, June 2010.
[4] J. Gu, D. Barker, and M. Pecht, "Uncertainty assessment of prognostics of electronics subject to random vibration," in Proceedings of the AAAI Fall Symposium on Artificial Intelligence for Prognostics, pp. 50-57, November 2007.

[5] D. Gucik-Derigny, A. Zolghadri, E. Suhir, and L. Bechou, "A model-based prognosis strategy for prediction of Remaining Useful Life of Ball-Grid-Array Interconnections," IFAC Proceedings, vol. 47, no. 3, pp. 7354-7360, 2014.

[6] R. Brincker, "Some elements of operational modal analysis," Shock and Vibration, vol. 2014, Article ID 325839, 11 pages, 2014.

[7] P. Mohanty and D. J. Rixen, "Identifying mode shapes and modal frequencies by operational modal analysis in the presence of harmonic excitation," Experimental Mechanics, vol. 45, no. 3, pp. 213-220, 2005.

[8] V. H. Vu, M. Thomas, A. A. Lakis, and L. Marcouiller, "Shorttime autoregressive (STAR) modeling for operational modal analysis of non-stationary vibration," in Vibration and Structural Acoustics Analysis, pp. 59-77, Springer, Berlin, Germany, 2011.

[9] D. Ning and Y. Gong, "Shocking fault component of abnormal sound signal in the fault engine extract method based on linear superposition method and cross-correlation analysis," Advances in Mechanical Engineering, vol. 7, no. 8, pp. 1-9, 2015.

[10] L. Soria, B. Peeters, J. Anthonis, and H. V. der Auweraer, "Operational Modal Analysis and the performance assessment of vehicle suspension systems," Shock and Vibration, vol. 19, no. 5, pp. 1099-1113, 2012.

[11] S.-D. Zhou, L. Liu, W. Yang, and Z.-S. Ma, "Operational modal identification of time-varying structures via a vector multistage recursive approach in hybrid time and frequency domain," Shock and Vibration, vol. 2015, Article ID 397364, 13 pages, 2015.

[12] B. Peeters, H. Van der Auweraer, F. Vanhollebeke, and P. Guillaume, "Operational modal analysis for estimating the 
dynamic properties of a stadium structure during a football game," Shock and Vibration, vol. 14, no. 4, pp. 283-303, 2007.

[13] M. J. Whelan, M. V. Gangone, K. D. Janoyan, and R. Jha, "Realtime wireless vibration monitoring for operational modal analysis of an integral abutment highway bridge," Engineering Structures, vol. 31, no. 10, pp. 2224-2235, 2009.

[14] A. Mironov, P. Doronkin, A. Priklonsky, and I. Kabashkin, "Condition monitoring of operating pipelines with operational modal analysis application," Transport and Telecommunication Journal, vol. 16, no. 4, pp. 305-319, 2015.

[15] D. Steinberg, Vibration Analysis for Electronic Equipment, A Wiley Inter-Science Publication, 3rd edition, 2000.

[16] Y. Chen, L. Yang, B. Liu, and D. Xue, "Applicability study of Steinberg vibration fatigue model in electronic products," in Proceedings of the Prognostics and System Health Management Conference (PHM-Hunan '14), pp. 31-35, Zhangiiaijie City, China, August 2014.

[17] F. Liu and G. Meng, "Random vibration reliability of BGA leadfree solder joint," Microelectronics Reliability, vol. 54, no. 1, pp. 226-232, 2014.

[18] S. Chesné and A. Deraemaeker, "Damage localization using transmissibility functions: a critical review," Mechanical Systems and Signal Processing, vol. 38, no. 2, pp. 569-584, 2013.

[19] G. Steenackers, C. Devriendt, and P. Guillaume, "On the use of transmissibility measurements for finite element model updating," Journal of Sound and Vibration, vol. 303, no. 3-5, pp. 707-722, 2007.

[20] J. H. Lim, "A correlation study of satellite finite element model for coupled load analysis using transmissibility with modified correlation measures," Aerospace Science and Technology, vol. 33, no. 1, pp. 82-91, 2014.

[21] L. Yang, Y. Chen, Z. Yuan, and L. Chen, "Effect of vibration transmissibility on fatigue lifetime of electronic devices," in Industrial Engineering, Management Science and Applications, pp. 1031-1039, Springer, Berlin, Germany, 2015.

[22] M. Vet, "Vibration analysis of thin rectangular plates," Machine Design, vol. 39, no. 9, pp. 193-202, 1967.

[23] T. Irvine, "Extending Steinberg's Fatigue Analysis of Electronics Equipment Methodology to a Full Relative Displacement vs. Cycles Curve, Revision C, Vibrationdata," 2013. 


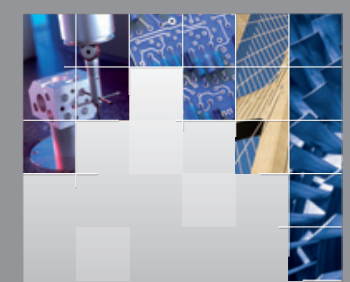

\section{Enfincering}
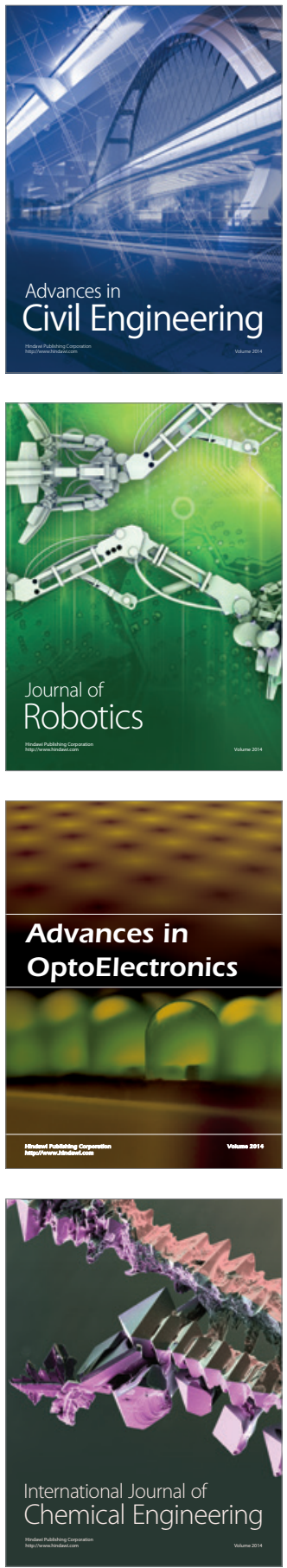

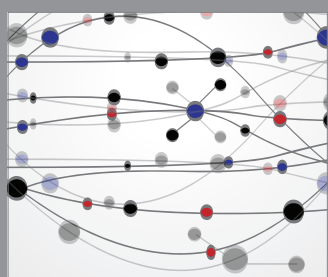

The Scientific World Journal

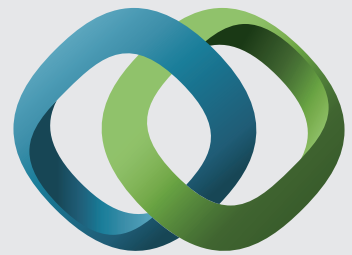

\section{Hindawi}

Submit your manuscripts at

http://www.hindawi.com
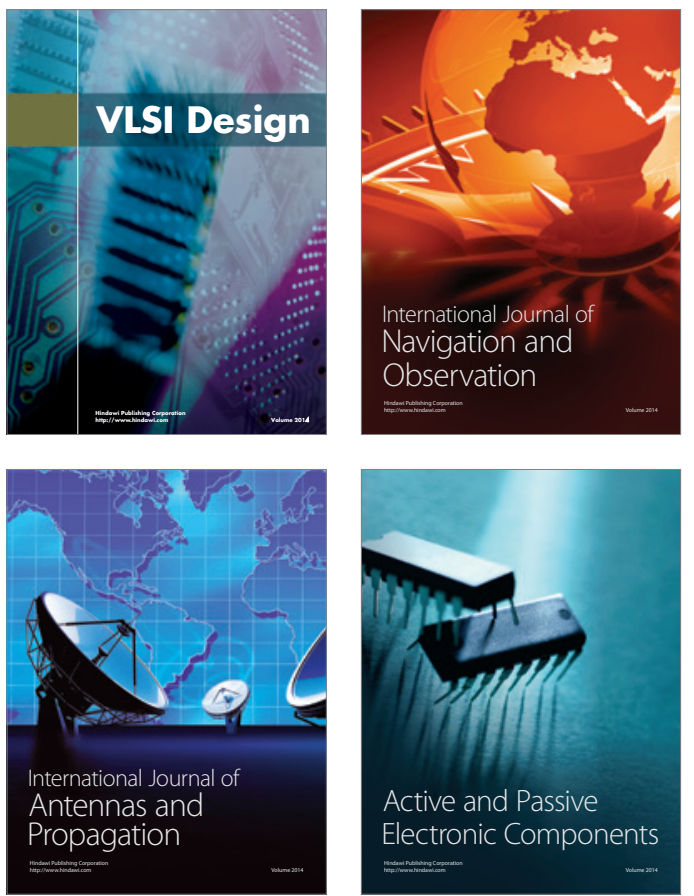
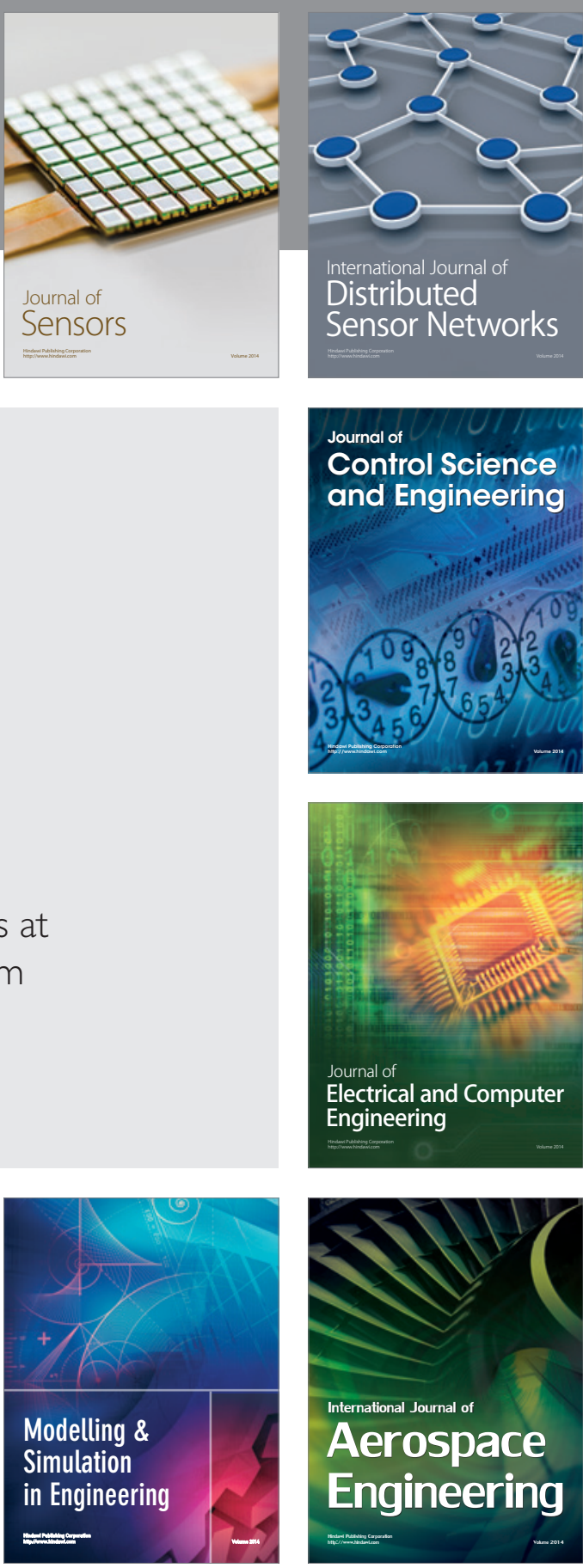

International Journal of

Distributed

Sensor Networks

Journal of

Control Science

and Engineering
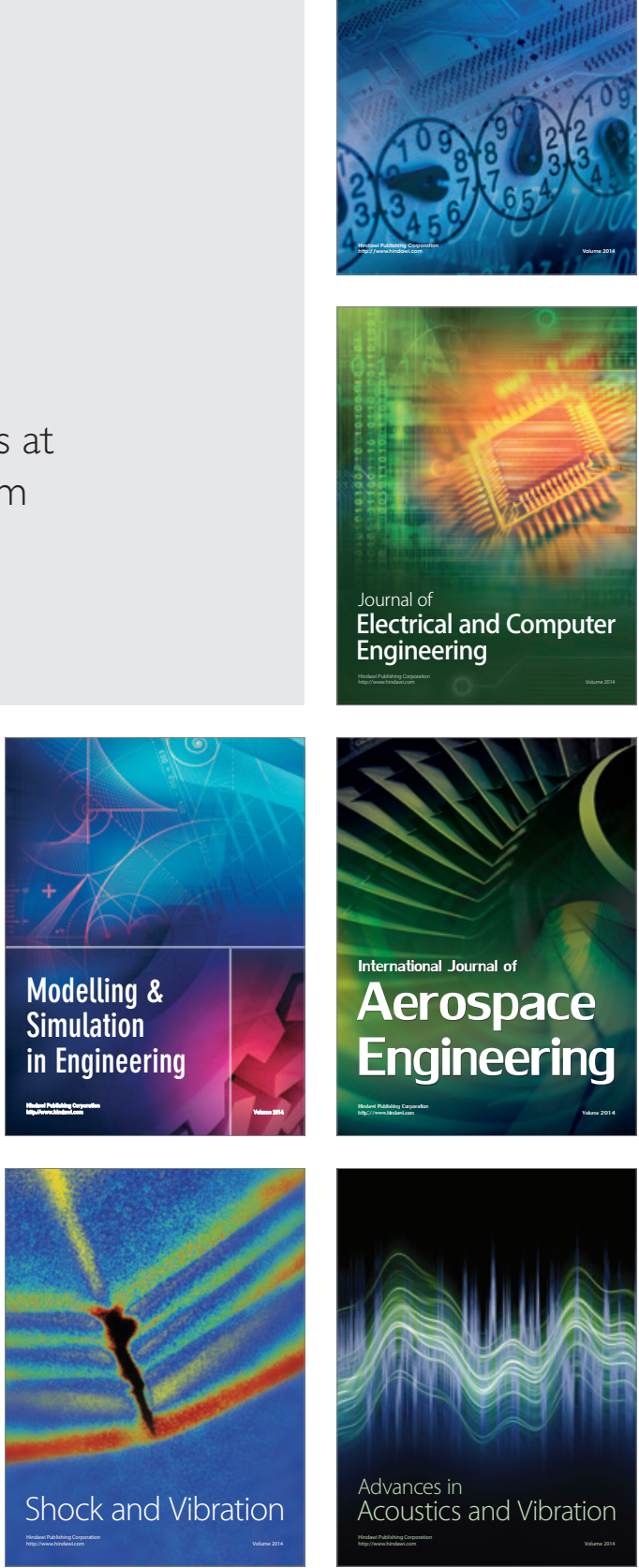